\title{
Variable Deceleration
}

National Cancer Institute

\section{Source}

National Cancer Institute. Variable Deceleration. NCI Thesaurus. Code C92914.

An abrupt decrease in the fetal heart rate below baseline that lasts 15 seconds to less

than two minutes. The decrease from baseline fetal heart rate is greater than or equal to 15 beats per minute, and the time from onset to nadir of the deceleration is less than 30 seconds. Variable fetal heart rate decelerations that are associated with successive uterine contractions commonly have varying onsets, depths, and durations. 